\title{
VARIABILIDAD MORFOLÓGICA DE FRUTOS DE PEUMO (Cryptocarya alba (Mol.) Looser) DE DISTINTAS LOCALIDADES DE LA REGIÓN DEL BIO BIO
}

Chung, Patricio"; Gutiérrez, Braulio"; Benedetti, Susana ${ }^{2}$

\section{RESUMEN}

Peumo (Cryptocarya alba (Mol.) Looser) es una especie endémica de Chile, perteneciente a la familia Lauraceae. Esta familia agrupa a numerosas especies distribuidas principalmente en las zonas tropicales y subtropicales del planeta. En el país peumo se distribuye entre las provincias del Limarí, región de Coquimbo, y Cautín, región de La Araucanía, desde la cordillera de la costa hasta la cordillera de Los Andes donde puede alcanzar altitudes de hasta $1500 \mathrm{msnm}$.

Su uso es amplio, se la emplea como planta medicinal para una serie de enfermedades; sus frutos son comestibles; su madera es utilizada como combustible en forma de leña o carbón, para la elaboración de parqué, utensilios para la agricultura, hormas para zapatos y tacos, y también se la usa como especie ornamental por su follaje y vistosos frutos rojos.

Se presenta una síntesis de un estudio de caracterización morfológica de frutos de peumo colectados en 10 localidades de la región del Biobío. Se entregan antecedentes de la variabilidad entre localidades para variables de tamaño, forma y peso de frutos completos de peumo, de sus semillas y de la pulpa de los mismos.

Se concluye que las variables están relacionadas entre sí, que existen diferencias estadísticamente significativas entre localidades, y que en general los frutos de mayor, tamaño, peso contenido de pulpa y peso de sus semillas son los correspondientes a la zona de Quillón.

Las implicancias de estos resultados pueden resultar de interés, como cuantificación inicial de la variabilidad de los frutos de peumo, para generar programas de selección y mejoramiento orientados a mejorar características particulares de los mismos, como es el grosor de la pulpa, el calibre de los frutos u otros relacionados, que tengan incidencia en la productividad y rendimiento de compuestos extraíbles de los frutos o en otros usos de los mismos.

Palabras clave: Peumo, Cryptocarya alba, variabilidad de frutos, región del Bio Bio.

\footnotetext{
${ }_{1}^{1}$ Instituto Forestal, sede Bio Bio. pchung@infor.cl; bgutierr@infor.cl

2 Instituto Forestal, sede Metropolitana. sbenedet@infor.cl
} 


\section{SUMMARY}

Peumo (Cryptocarya alba (Mol.) Looser) is a Chilean endemic tree of the Lauraceae family which includes a number of species over the world tropical and subtropical areas. Its natural distribution covers areas between the Limarí province, Coquimbo region, to the Cautín province, Araucanía region, from the coastal zones to the Andes ranges where the species can reach up to 1,500 masl.

The species has a wide range of uses; medicinal plant, edible fruits, fuelwood and charcoal; wood for parquet, agricultural tools and shoe moulds, and ornamental uses due to its foliage and attractive red fruits.

Results of a morphological study on the Peumo fruits at de Bio Bio region are presented. Fruits were collected from ten provenances in the region and information on fruits, pulp and seeds variability in size, form and weight is analysed.

It is concluded that those variables are related between them, significant differences between provenances are found and the higher values on fruits size, weight and pulp contents, as well as on its seeds weight, correspond to the Quillón provenances.

The found variability on the fruits could be of interests as a first approach to generate selection and improvement programmes oriented to better particular characteristics, such as pulp thickness, fruit size or others, which can improve the yield and productivity of removable compounds from the fruits or other uses of them.

Key Words: Peumo, Cryptocarya alba, Fruits variabilty, Bio Bio Region 


\section{INTRODUCCIÓN}

Los bosques naturales de la región del Bio Bio presentan un alto potencial para desarrollo económico, social y ambiental. Entre los productos asociados a estos bosques resaltan los frutos silvestres, los cuales poseen múltiples aplicaciones y usos. Entre tales frutos destacan particularmente los de peumo, respecto de los cuales se registran diversos usos y conocimientos tradicionales que no están suficientemente difundidos ni aprovechados, valorándose a la especie fundamentalmente por su uso como fuente de leña y carbón.

Peumo es una especie que tiene distintos usos en el ámbito rural, uno de ellos es el consumo de sus frutos. Los frutos de esta especie son aromáticos, comestibles y presentan principios antioxidantes. Los frutos también se han utilizado en gastronomía para la fabricación de postres (Boragó, 2011). Por su parte, las hojas se utilizan como infusión en enfermedades hepáticas (Montes, 1987), en hemorragias y reumatismos (Vogel et al., 2008). Con las semillas molidas se preparan pomadas para el tratamiento de afecciones ginecológicas y para dolencias abdominales derivadas de enfriamientos (Ibaca, 2001), atribuyéndoseles también propiedades para aliviar los dolores articulares y musculares cuando se aplican en forma de compresas (Barreau y Salas, 2009). Las propiedades medicinales de esta planta se pueden explicar por la presencia del tanino, que posee cualidades astringentes, y por el aceite esencial que contienen

En tal contexto, existe el interés y el desafío por desarrollar técnicas adecuadas y amigables con el medio ambiente que permitan producir y aprovechar sustentablemente los frutos de peumo, generando productos diversificados, de buena calidad y con un stock permanente, que los lleve a obtener mayor valor agregado y asegure su permanencia en los mercados nacionales e internacionales. Para tal efecto, así como para iniciar el mejoramiento genético de los atributos de interés de los frutos de peumo, se requiere conocer información respecto de su variabilidad. Por lo mismo, en este documento se realiza una caracterización y evaluación de la variabilidad morfológica exhibida por los frutos de esta especie, en diferentes procedencias de su distribución natural en la región del Bio Bio.

\section{MATERIAL Y MÉTODO}

Se realizó una búsqueda de especímenes adultos de peumo, en etapa de fructificación, con el propósito de extraer manualmente muestras de frutos para los análisis respectivos en laboratorio. Una vez seleccionado los árboles y colectados los frutos, se registro información dasométrica del árbol (altura, diámetro) y características del punto de muestreo (coordenadas, altitud, exposición tipo de suelo, posición fisiográfica) (Cuadro N 1).

Cuadro $\mathrm{N}^{\circ} 1$

CARACTERIZACIÓN DE SITIOS PARA LA OBTENCIÓN DE MUESTRAS DE FRUTOS

\begin{tabular}{|c|c|c|c|c|c|c|c|c|c|}
\hline \multirow{2}{*}{ Origen } & \multirow{2}{*}{ Sector } & \multicolumn{2}{|c|}{ Coordenadas } & \multirow{2}{*}{$\begin{array}{l}\text { Altitud } \\
\text { (msnm) }\end{array}$} & \multirow{2}{*}{$\begin{array}{c}\text { Altura } \\
\text { (m) }\end{array}$} & \multirow{2}{*}{$\begin{array}{l}\text { Diámetro } \\
(\mathrm{cm})\end{array}$} & \multirow{2}{*}{ Tipo de Suelo } & \multirow{2}{*}{ Exposición } & \multirow{2}{*}{ Ubicación } \\
\hline & & Latitud & Longitud & & & & & & \\
\hline 1 & Colico Km 7,5 & $37^{\circ} 21^{\prime} 00^{\prime \prime} \mathrm{S}$ & $72^{\circ} 50^{\prime} 58^{\prime \prime} 0$ & 105 & 16 & 29 & Arcilloso & SE & Fondo Ladera Camino \\
\hline 2 & Nacimiento-Los Patos 1 & $37^{\circ} 27^{\prime} 42^{\prime \prime} \mathrm{S}$ & $72^{\circ} 55^{\prime} 41^{\prime \prime} 0$ & 340 & 13 & 45 & Limo arcilloso & SE & Deslinde Parcela \\
\hline 3 & Nacimiento-Los Patos 2 & $37^{\circ} 27^{\prime} 42^{\prime \prime} \mathrm{S}$ & $72^{\circ} 55^{\prime} 41^{\prime \prime} 0$ & 340 & 15 & 26,8 & Limo arcilloso & SE & Deslinde Parcela \\
\hline 4 & Quilleco & $37^{\circ} 28^{\prime} 14^{\prime \prime} \mathrm{S}$ & $77^{\circ} 58^{\prime} 49^{\prime \prime} 0$ & 335 & 8 & 18 & Trumao & Plano & Plaza Ciudad \\
\hline 5 & Quillón-1 & $36^{\circ} 44^{\prime} 32^{\prime \prime} \mathrm{S}$ & $72^{\circ} 28^{\prime} 04^{\prime \prime} 0$ & 60 & 14 & 38,4 & Limo arcilloso & Plano & Plazoleta Ciudad \\
\hline 6 & Quillón-2 & $36^{\circ} 44^{\prime} 32^{\prime \prime} \mathrm{S}$ & $72^{\circ} 28^{\prime} 04^{\prime \prime} 0$ & 60 & 8 & 21,6 & Limo arcilloso & Plano & Plazoleta Ciudad \\
\hline 7 & Parque Cuyanmahuida & $36^{\circ} 49^{\prime} 32^{\prime \prime} \mathrm{S}$ & $72^{\circ} 43^{\prime} 52^{\prime \prime} 0$ & 228 & 10 & 14 & Arcilloso & SE & Fondo de Ladera \\
\hline 8 & Collico Km 50-1 & $36^{\circ} 49^{\prime} 20^{\prime \prime} \mathrm{S}$ & $72^{\circ} 44^{\prime} 31^{\prime \prime} 0$ & 190 & 13 & 15 & Limo arcilloso & NE & Media Ladera Camino \\
\hline 9 & Collico Km 50-2 & $36^{\circ} 49^{\prime} 20^{\prime \prime} \mathrm{S}$ & $72^{\circ} 44^{\prime} 31^{\prime \prime} 0$ & 177 & 12 & 20 & Limo arcilloso & SE & Ladera Camino \\
\hline 10 & Los Patos-Cipriano & $37^{\circ} 27^{\prime} 42^{\prime \prime} \mathrm{S}$ & $72^{\circ} 55^{\prime} 41^{\prime \prime} 0$ & 320 & 7 & 15 & Arcilloso & Plano & Deslinde Parcela \\
\hline
\end{tabular}


Para efectos de transporte, cada muestra se envasó en bolsas de papel para evitar la condensación y posterior proceso de pudrición de los frutos. Cada bolsa fue identificada con el código de registro tomado en terreno, para posteriormente colocarlas en un contenedor sellado a una temperatura de $3-5^{\circ} \mathrm{C}$ y finalmente transportarlas a laboratorio para su almacenamiento, procesamiento y evaluación.

A cada una de las muestras se le calcularon los siguientes parámetros: Peso húmedo y peso seco del fruto, la semilla y la pulpa; largo y ancho del fruto; y espesor de la pulpa (Figuras $\mathrm{N}^{\circ}$ $1,2$ y 3$)$.

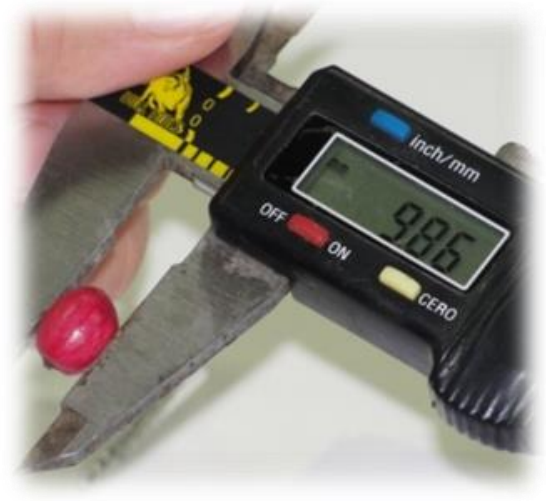

Figura $\mathrm{N}^{\circ} 1$

MEDICIÓN DEL ANCHO DEL FRUTO

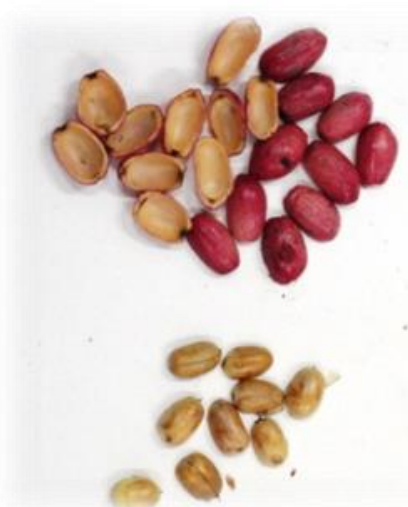

Figura $\mathrm{N}^{\circ} 2$ ASPECTO DE LA SEMILLA Y PULPA

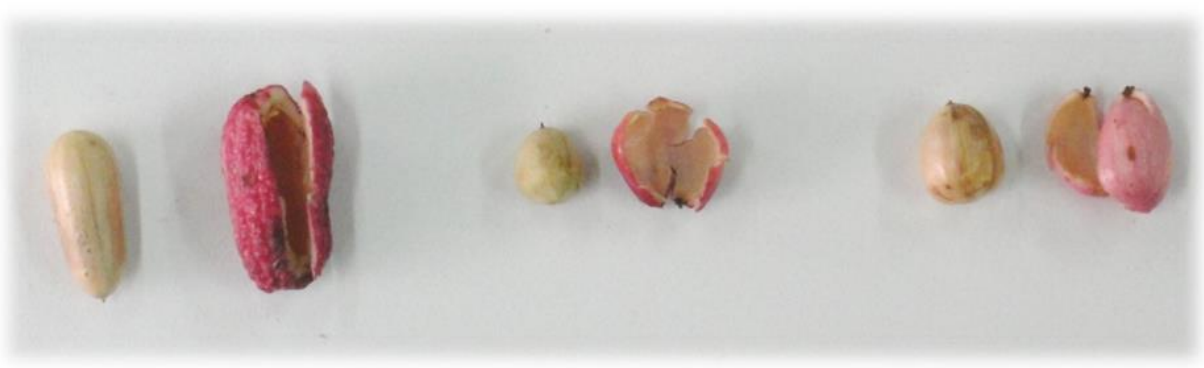

Figura $\mathrm{N}^{\circ} 3$

VARIABILIDAD DE FRUTOS EN TAMAÑO Y FORMA DE LAS SEMILLAS Y GROSOR DE LA PULPA

Para la medición del largo y ancho de los frutos, así como para el espesor de pulpa se utilizó un pie de metro digital, realizándose varias mediciones bajo distintos ejes, para luego obtener una medición promedio, evitando o minimizando así errores por algunas deformaciones particulares del fruto.

En relación al secado de las muestras, se utilizó una estufa automática, dejando las muestras envueltas en papel de aluminio a una temperatura de $80^{\circ} \mathrm{C}$ por 48 horas, para luego ser pesadas en una balanza de precisión. 
Con las variables indicadas se construyeron índices que explican la variabilidad observada en mejor forma que las variables individuales por si solas. Los índices construidos fueron: Índice de forma (Largo del fruto/ancho del fruto), índice de humedad del fruto (peso húmedo/peso seco del fruto), índice de humedad de la semilla (peso húmedo/peso seco de la semilla), índice de humedad de la pulpa (peso húmedo/peso seco de la pulpa).

Sede Biobío.

Las mediciones se llevaron a cabo en el Laboratorio de Micología del Instituto Forestal,

Los datos obtenidos de las variables e índices se analizaron estadísticamente utilizando para ello el software estadístico INFOSTAT ${ }^{\circledR}$.

\section{RESULTADOS Y DISCUSIÓN}

\section{Índice de Forma del Fruto}

El índice de forma es un indicador de la relación entre el largo y ancho de los frutos, donde valores más altos indican frutos de forma más alargada, en tanto que valores más bajos son indicativos de frutos más cortos o redondeados.

En el Cuadro $N^{\circ} 2$ se muestra la diferenciación estadística entre los orígenes evaluados, observándose la formación de 5 grupos con diferencias significativas entre ellos. Lo anterior indica una importante variación entre procedencias, en términos de tamaño (largo y ancho de frutos) y forma de los mismos (índice de forma).

Destacan con el mayor tamaño y forma más alargada los frutos del origen Quillón 2. En la Figura $N^{\circ} 4$ se muestra fotografía que ilustra la variación en tamaño y forma de los frutos.

En términos de tamaño, los frutos más grandes en largo y ancho son los de las procedencias Quillón-1 y Quillón-2, los que también resultan ser los de mayor peso (Cuadro N³).

\section{Cuadro $\mathrm{N}^{\circ} 2$}

LARGO, ANCHO E ÍNDICE DE FORMA DE FRUTOS DE DISTINTAS PROCEDENCIAS

\begin{tabular}{|l|c|cc|cc|rc|}
\hline Procedencia & $\mathbf{n}$ & \multicolumn{2}{|c|}{$\begin{array}{c}\text { (a) } \\
\text { (argo }\end{array}$} & $\begin{array}{c}\text { (b) } \\
\text { Ancho } \\
(\mathbf{m m})\end{array}$ & $\begin{array}{c}\text { Índice de Forma } \\
(\mathbf{a} / \mathbf{b})\end{array}$ \\
\hline Nacimiento-Los Patos 1 & 30 & 16,61 & $\mathbf{b}$ & 12,32 & $\mathbf{f}$ & 1,35 & $\mathbf{a}$ \\
\hline Collico Km 50-2 & 30 & 13,50 & $\mathbf{a}$ & 9,89 & $\mathbf{b}$ & 1,37 & $\mathbf{a}$ \\
\hline Los Patos-Cipriano & 30 & 16,72 & $\mathbf{b}$ & 12,08 & $\mathbf{f}$ & 1,39 & $\mathbf{a}$ \\
\hline Colico Km 7,5 & 30 & 14,04 & $\mathbf{a}$ & 9,52 & $\mathbf{a}$ & 1,48 & $\mathbf{b}$ \\
\hline Collico Km 50-1 & 30 & 17,69 & $\mathbf{c}$ & 11,91 & $\mathbf{f}$ & 1,49 & $\mathbf{b}$ \\
\hline Nacimiento-Los Patos 2 & 30 & 17,41 & $\mathbf{c}$ & 10,72 & $\mathbf{c}$ & 1,63 & $\mathbf{c}$ \\
\hline Parque Cuyanmahuida & 30 & 18,24 & $\mathbf{d}$ & 11,13 & $\mathbf{d}$ & 1,64 & $\mathbf{c}$ \\
\hline Quilleco & 30 & 19,18 & $\mathbf{e}$ & 11,60 & $\mathbf{e}$ & 1,66 & $\mathbf{c}$ \\
\hline Quillón-1 & 30 & 22,48 & $\mathbf{f}$ & 12,09 & $\mathbf{f}$ & 1,86 & $\mathbf{d}$ \\
\hline Quillón-2 & 30 & 24,45 & $\mathbf{g}$ & 12,08 & $\mathbf{f}$ & 2,03 & $\mathbf{e}$ \\
\hline
\end{tabular}

Medias con una letra común en la misma columna no son significativamente diferentes (Scott y Knott, $p<=0,05$ ) 


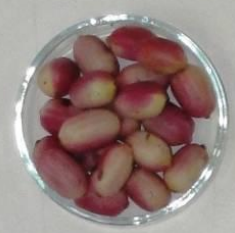

SECTOR 1

Colico Alto

Región del Bio Bio

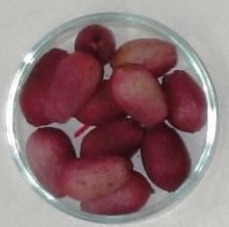

\section{SECTOR 4}

Quilleco

Región del Bio Bio

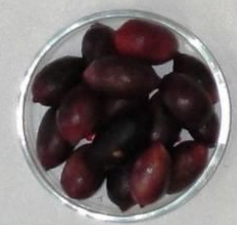

SECTOR 7

Parque Coyanmahuida Región del Bio Bio

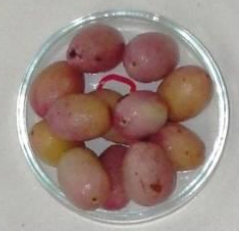

SECTOR 2

Los Patos 1

Región del Bio Bio

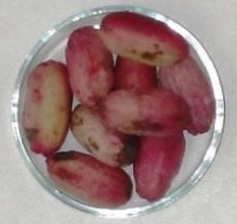

\section{SECTOR 5}

Quillón 1

Región del Bio Bio

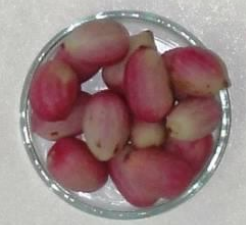

SECTOR 8

Curva Collico 1

Región del Bio Bio

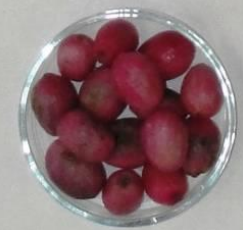

\section{SECTOR 10}

Cipriano

Región del Bio Bio

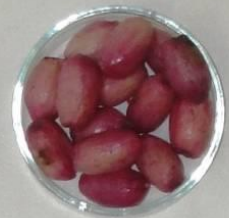

SECTOR 3

Los Patos 2

Región del Bio Bio

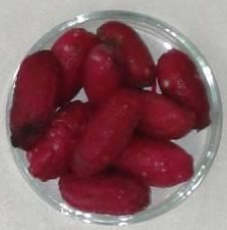

\section{SECTOR 6}

Quillón 2

Región del Bio Bio

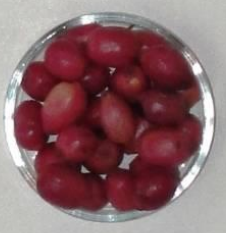

\section{SECTOR 9}

Curva Collico 2

Región del Bio Bio

Figura $N^{\circ} 4$

ASPECTO GENERAL DE LA VARIACIÓN EN TAMAÑO Y FORMA DE FRUTOS DE DISTINTAS PROCEDENCIAS 


\section{Índice de Humedad del Fruto}

El índice de humedad de los frutos presenta una variación significativa entre las procedencias evaluadas (Cuadro $\mathrm{N}^{\circ} 3$ ). Valores altos en este índice indican mayor contenido de humedad en los frutos antes de su secado, mientras que valores cercanos a uno indican que los frutos posen un contenido de humedad muy bajo.

En términos de porcentaje de contenido de humedad los frutos de peumo varían entre 40 y $51 \%$, pero para efectos de diferenciación estadística se utilizó el índice de humedad en vez del contenido de humedad en porcentaje. En cuanto a peso seco y peso húmedo, los mayores valores corresponden a los frutos de las procedencias Quillón-1 y Quillón-2.

El peso húmedo de los frutos es muy importante al influir en la producción de plantas en vivero, por estar directamente relacionado con el número de semillas por kilógramo. Este dato permite la planificación en vivero, al conocerse la cantidad por procedencia para la producción programada. Si se toma el peso húmedo de las semillas, los orígenes que presenta menos semillas por kilógramo son los obtenidos desde la localidad de Quillón (Quillón-2 y Quillón-1), por cuanto sus frutos son más grandes y pesados; mientras que la que posee la mayor cantidad de semillas por kilogramo es la originaria de la localidad de Colico (Colico km 7,5), con frutos más pequeños y livianos.

Este índice de humedad podría ayudar en futuros trabajos que tengan relación con la extracción de elementos nutricionales y/o medicinales desde los frutos de peumo. Estudios tendientes a determinar la relación entre el grado de madurez de los frutos y la magnitud del índice calculado, podrían tener una relación significativa con las magnitudes en las concentraciones de ciertos elementos de importancia que pudieran contener los frutos, como son las concentraciones de vitaminas o ácidos grasos, entre otros, para un origen o individuo determinado.

Cuadro $\mathrm{N}^{\circ} 3$

PESOS HÚMEDO, PESO SECO E ÍNDICE DE HUMEDAD DE FRUTOS DE DISTINTAS PROCEDENCIAS

\begin{tabular}{|c|c|c|c|c|c|c|c|c|c|}
\hline Sector & $\mathbf{n}$ & $\begin{array}{r}\text { (a } \\
\text { Pe } \\
\text { Húm } \\
(\mathrm{g}\end{array}$ & & $\begin{array}{r}(\mathrm{b} \\
\mathrm{Pe} \\
\mathrm{Se} \\
(\mathrm{g}\end{array}$ & & $\begin{array}{l}\text { Índic } \\
\text { hum } \\
(a)\end{array}$ & & $\begin{array}{l}\text { Frutos } \\
(\mathrm{N} / \mathrm{kg})\end{array}$ & $\begin{array}{c}\text { Contenido de } \\
\text { Humedad } \\
(\%)\end{array}$ \\
\hline Collico Km 50-1 & 30 & 1,64 & d & 0,97 & d & 1,69 & $\mathbf{a}$ & 610 & 40,9 \\
\hline Collico Km 50-2 & 30 & 0.90 & a & 0,53 & $\mathbf{a}$ & 1,72 & a & 1111 & 41,6 \\
\hline Parque Cuyanmahuida & 30 & 1,46 & c & 0,85 & C & 1,73 & $\mathbf{a}$ & 685 & 41,7 \\
\hline Los Patos-Cipriano & 30 & 1,54 & c & 0,90 & d & 1,73 & $\mathbf{a}$ & 649 & 41,8 \\
\hline Colico Km 7,5 & 30 & 0,85 & $\mathbf{a}$ & 0,49 & $\mathbf{a}$ & 1,74 & $\mathbf{a}$ & 1176 & 42,4 \\
\hline Quilleco & 30 & 1,74 & d & 0,96 & d & 1,82 & b & 575 & 44,5 \\
\hline Nacimiento-Los Patos 1 & 30 & 1,52 & c & 0,80 & c & 1,90 & c & 658 & 47,2 \\
\hline Quillón-2 & 30 & 2,33 & $f$ & 1,19 & $\mathbf{f}$ & 1,96 & c & 429 & 48,9 \\
\hline Quillón-1 & 30 & 2,11 & e & 1,03 & e & 2,06 & d & 474 & 51,0 \\
\hline Nacimiento-Los Patos 2 & 30 & 1,35 & b & 0,66 & b & 2,06 & d & 741 & 51,2 \\
\hline
\end{tabular}

Medias con una letra común en la misma columna no son significativamente diferentes (Scott y Knott, $p<=0,05$ )

\section{Índice de humedad de la Semilla}

El análisis estadístico del índice de humedad de la semilla permite segregar dos grupos de procedencias con diferencias estadísticamente significativas (Cuadro $\mathrm{N}^{\circ} 4$ ).

En términos de peso seco, los mayores valores corresponden a las semillas de las procedencias Quillón-2, Collico-1, Los Patos y Parque Cuyanmahuida. 
Cuadro $\mathrm{N}^{\circ} 4$

PESO HÚMEDO, PESO SECO E ÍNDICE DE HUMEDAD DE SEMILLAS DE DISTINTAS PROCEDENCIAS

\begin{tabular}{|c|c|c|c|c|c|c|c|c|c|}
\hline Origen & $\mathbf{n}$ & $\begin{array}{r}\text { (a } \\
\text { Pes } \\
\text { Húm } \\
\text { (g }\end{array}$ & & $\begin{array}{r}\text { (b } \\
\text { Peso } \\
\text { (g }\end{array}$ & & $\begin{array}{l}\text { Índice } \\
\text { Hume } \\
(a / \mathrm{t}\end{array}$ & & $\begin{array}{l}\text { Semillas } \\
(\mathrm{N} / \mathrm{kg})\end{array}$ & $\begin{array}{c}\text { Contenido } \\
\text { de } \\
\text { Humedad } \\
(\%)\end{array}$ \\
\hline Los Patos-Cipriano & 30 & 1,17 & c & 0,74 & d & 1,59 & a & 855 & 36,9 \\
\hline Parque Cuyanmahuida & 30 & 1,29 & C & 0,80 & d & 1,61 & a & 775 & 38,0 \\
\hline Colico Km 7,5 & 30 & 0,68 & a & 0,42 & a & 1,63 & $\mathbf{a}$ & 1471 & 36,9 \\
\hline Quilleco & 30 & 1,08 & b & 0,67 & c & 1,63 & $\mathbf{a}$ & 926 & 37,3 \\
\hline Collico Km 50-1 & 30 & 1,25 & c & 0,75 & d & 1,67 & $\mathbf{a}$ & 800 & 40,0 \\
\hline Collico Km 50-2 & 30 & 0,60 & a & 0,36 & $\mathbf{a}$ & 1,70 & b & 1667 & 40,9 \\
\hline Nacimiento-Los Patos 2 & 30 & 1,10 & b & 0,64 & c & 1,73 & b & 909 & 42,1 \\
\hline Quillón-2 & 30 & 1,35 & c & 0,77 & d & 1,75 & b & 741 & 42,6 \\
\hline Quillón-1 & 30 & 1,22 & c & 0,69 & c & 1,77 & b & 820 & 43,2 \\
\hline Nacimiento-Los Patos 1 & 30 & 0,98 & b & 0,55 & b & 1,79 & b & 1020 & 44,1 \\
\hline
\end{tabular}

Medias con una letra común en la misma columna no son significativamente diferentes (Scott y Knott, $p<=0,05$ )

\section{Índice de humedad de la Pulpa}

Los análisis de peso de la pulpa indican que los orígenes Quillón-1, Quillón-2 y Quilleco, son las que presentan el mayor peso seco en pulpa, lo que indica una condición que podría ser beneficiosa para el uso de los frutos como elemento de trabajo en el área nutricional y de su uso como alimento (Cuadro $\mathrm{N}^{\circ} 5$ ). Estas mismas procedencias presentan también el mayor peso húmedo

\section{Cuadro $\mathrm{N}^{\circ} 5$}

PESO HÚMEDO, PESO SECO E ÍNDICE DE HUMEDAD DE LA PULPA DE FRUTOS DE DISTINTAS PROCEDENCIAS

\begin{tabular}{|c|c|c|c|c|c|c|c|c|}
\hline \multirow{2}{*}{$\begin{array}{l}\text { Origen } \\
\text { Quillón-2 }\end{array}$} & \multirow{2}{*}{$\begin{array}{l}\mathbf{n} \\
30\end{array}$} & \multicolumn{2}{|c|}{$\begin{array}{c}\text { (a) } \\
\text { Peso } \\
\text { Húmedo } \\
\text { (g) }\end{array}$} & \multicolumn{2}{|c|}{$\begin{array}{c}\text { (b) } \\
\text { Peso Seco } \\
\text { (g) }\end{array}$} & \multicolumn{2}{|c|}{$\begin{array}{l}\text { Índice de } \\
\text { humedad } \\
(a / b)\end{array}$} & \multirow{2}{*}{$\begin{array}{c}\begin{array}{c}\text { Contenido } \\
\text { de } \\
\text { Humedad } \\
\text { (\%) }\end{array} \\
37,38\end{array}$} \\
\hline & & 0,91 & d & 0,57 & e & 1,61 & $\mathbf{a}$ & \\
\hline Los Patos-Cipriano & 30 & 0,26 & a & 0,17 & b & 1,70 & $\mathbf{a}$ & 32,65 \\
\hline Nacimiento-Los Patos 2 & 30 & 0,22 & a & 0,14 & b & 1,71 & $\mathbf{a}$ & 39,24 \\
\hline Collico Km 50-2 & 30 & 0,23 & a & 0,14 & b & 1,74 & $\mathbf{a}$ & 39,76 \\
\hline Collico Km 50-1 & 30 & 0,30 & a & 0,16 & b & 1,94 & a & 48,15 \\
\hline Quilleco & 30 & 0,79 & c & 0,39 & d & 2,04 & $\mathbf{a}$ & 50,59 \\
\hline Colico Km 7,5 & 30 & 0,18 & a & 0,08 & $\mathbf{a}$ & 2,17 & $\mathbf{a}$ & 53,23 \\
\hline Nacimiento-Los Patos 1 & 30 & 0,61 & b & 0,28 & c & 2,22 & $\mathbf{a}$ & 54,92 \\
\hline Quillón-1 & 30 & 0,99 & d & 0,37 & d & 2,66 & b & 62,22 \\
\hline Parque Cuyanmahuida & 30 & 0,22 & a & 0,07 & $\mathbf{a}$ & 3,57 & C & 67,61 \\
\hline
\end{tabular}

Medias con letra común en la misma columna no son significativamente diferentes (Scott y Knott, $p<=0,05$ ) 


\section{Espesor de la Pulpa}

El análisis del espesor de pulpa arroja tres orígenes principales, en relación a la cantidad de pulpa que contienen los frutos, estos son en orden descendente Quillón-2, Quillón-1 y Quilleco (Cuadro $\mathrm{N}^{\circ} 6$ ). Estas tres difieren en forma significativa frente a los otros orígenes, pudiendo a través de estas iniciarse un proceso de selección y mejoramiento en relación a aumentar el contenido de pulpa en frutos de peumo.

Además, lo anterior deberá ser complementado con las características nutricionales y/o medicinales que se quiera mejorar o aumentar, pudiendo incluir a algunas otras procedencias que posean una mayor presencia de la características que se desea mejorar y con ello poder combinar a través de un programa de hibridación para la especie.

Cuadro $\mathrm{N}^{\circ} 6$ ESPESOR DE PULPA DE FRUTOS DE DISTINTAS PROCEDENCIAS

\begin{tabular}{|l|c|cc|}
\hline \multicolumn{1}{|c|}{ Origen } & n & \multicolumn{2}{c|}{$\begin{array}{c}\text { Medias } \\
\text { (mm) }\end{array}$} \\
\hline Collico Km 50-1 & 30 & 0,35 & a \\
\hline Colico Km 7,5 & 30 & 0,36 & a \\
\hline Parque Cuyanmahuida & 30 & 0,42 & a \\
\hline Collico Km 50-2 & 30 & 0,45 & a \\
\hline Los Patos-Cipriano & 30 & 0,59 & b \\
\hline Nacimiento-Los Patos 1 & 30 & 0,92 & c \\
\hline Nacimiento-Los Patos 2 & 30 & 0,96 & c \\
\hline Quilleco & 30 & 1,15 & d \\
\hline Quillón-1 & 30 & 1,18 & d \\
\hline Quillón-2 & 30 & 1,25 & d \\
\hline
\end{tabular}

Medias con letra común en la misma columna no son significativamente diferentes (Scott y Knott, $p<=0,05$ )

\section{Comparación Simultánea de las Principales Variables Evaluadas}

Aun cuando no se determinaron coeficientes de correlación, resulta evidente que las variables evaluadas están altamente correlacionadas entre sí, resultando natural que los frutos más grandes sean su vez los más pesados, y que los frutos de mayor envergadura posean mayor cantidad de pulpa tanto en peso como en espesor, así como semillas de mayor peso que las de los frutos más pequeños.

En la Figura $N^{\circ} 5$ se resume la variación expresada por los frutos de los diez orígenes considerados en la evaluación, destacándose a los procedentes de la zona de Quillón como los de mayor tamaño, peso y contenido de pulpa. 


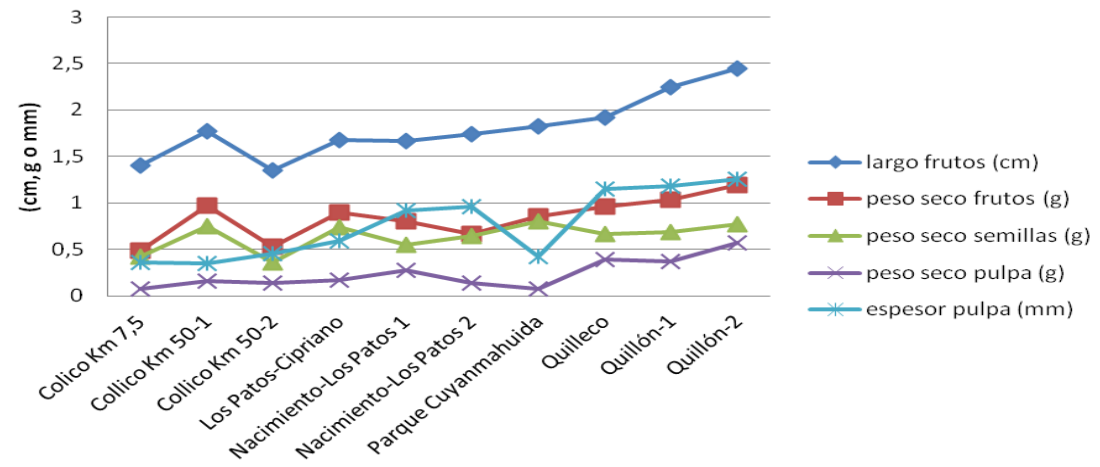

Figura $N^{\circ} 5$

VARIACIÓN DE FRUTOS DE DISTINTAS PROCEDENCIAS

\section{CONCLUSIONES}

Se confirma una gran variabilidad de los frutos de distintos orígenes respecto a forma, espesor de pulpa, tamaño del fruto, además de la relación peso húmedo/peso seco en relación al fruto, semilla y pulpa.

Los orígenes que más destacan tanto desde el punto de vista del tamaño como del de la cantidad de pulpa del fruto son Quillón-2, Quillón-1 y Quilleco. Este material podría ser el inicio, junto con una mayor selección de otros individuos, para un programa de mejoramiento genético que busque propiciar el aumento de una o varias características particulares que se quieran mejorar en plantas de peumo, pudiendo buscar objetivos como su uso como alimento, medicinal o la producción de sustancia químicas para usos diversos.

De los estudios realizados, resalta la necesidad de realizar otros estudios que consideren los aspectos nutricionales y químicos en relación al tipo de fruto y origen de estos. Por otro lado, la potencialidad de peumo como una especie fruto forestal deberá ser analizada, con el propósito de establecer futuros programas de mejoramiento, que implique el desarrollo del fruto como producto alimenticio y la posible extracción y elaboración de productos de tipo medicinal.

Las variaciones que se presentaron entre los orígenes de los frutos, para una pequeña muestra dentro de la extensa distribución de esta especie, lleva a pensar que existiría una gran variabilidad en torno a las poblaciones existentes en el país. La diversidad de situaciones de suelo y clima refleja una alta diversidad poblacional y posiblemente de individuos, que bajo trabajos de selección y mejoramiento pudieran presentar ciertas ventajas comparativas entre individuos para objetivos específicos de uso de la especie.

\section{REFERENCIAS}

Barreau, A. y Salas, V., 2009. Plantas que Curan, Compartiendo Vivencias y Saberes. Medicina Campesina de la Región del Bio Bio. TAC- Forestal Mininco. 73 p.

Boragò Restaurant, 2011. Endémica. http://www.borago.cl/blog/category/chile (obtenido el 23 de agosto de 2012).

Ibaca, R., 2001. Monografía de Árboles y Arbustos Chilenos con Propiedades Medicinales Aromáticas. Memoria para optar al título de Ingeniero Forestal. Universidad de Concepción, Facultad de Ciencias Forestales, Departamento de Silvicultura. 246 p. 
Montes, M., 1987. Aspectos de la Medicación Popular en la Región del Bio Bio, Chile. Acta Farm. Bonaerense. 6(2): 115-24.

Vogel, H.; Razmilic, I.; San Martín, J.; Doll, U. y González, B., 2008. Plantas Medicinales Chilenas. Experiencia de Domesticación y Cultivo de Boldo, Matico, Bailahuén, Canelo, Peumo y Maqui. Segunda Edición. Editorial de la Universidad de Talca. 194 p. 
